\title{
Protection of flunarizine on cerebral mitochondria injury induced by cortical spreading depression under hypoxic conditions
}

\author{
Fengpeng Li • Enchao Qiu · Zhao Dong • \\ Ruozhuo Liu $\cdot$ Shiwen Wu Shengyuan Yu
}

Received: 2 September 2010/Accepted: 25 November 2010/Published online: 25 February 2011

(C) The Author(s) 2011. This article is published with open access at Springerlink.com

\begin{abstract}
A rat cortical spreading depression (CSD) model was established to explore whether cerebral mitochondria injury was induced by CSD under both normoxic and hypoxic conditions and whether flunarizine had a protective effect on cerebral mitochondria. SD rats, which were divided into seven groups, received treatment as follows: no intervention (control Group I); $1 \mathrm{M} \mathrm{NaCl}$ injections (Group II); $1 \mathrm{M} \mathrm{KCl}$ injections (Group III); intraperitoneal flunarizine $(3 \mathrm{mg} / \mathrm{kg}) 30 \mathrm{~min}$ before $\mathrm{KCl}$ injections (Group IV); $14 \% \quad \mathrm{O}_{2}$ inhalation before $\mathrm{NaCl}$ injections (Group V); $14 \% \mathrm{O}_{2}$ inhalation followed by $\mathrm{KCl}$ injections (Group VI); $14 \% \mathrm{O}_{2}$ inhalation and intraperitoneal flunarizine followed by $\mathrm{KCl}$ injections (Group VII). Following treatment, brains were removed for the analysis of mitochondria transmembrane potential (MMP) and oxidative respiratory function after recording the number, amplitude and duration of CSD. The duration of CSD was significantly longer in Group VI than that in Group III. The number and duration of CSD in Group VII was significantly lower than that in Group VI. MMP in Group VI was significantly lower than that in Group III, and MMP in Group VII was significantly higher than that in Group VI. State 4 respiration in Group VI was significantly higher than that in Group III, and state 3 respiration in Group VII was significantly higher than that in Group VI. Respiration control of rate in Group VII was also significantly higher than that in Group VI. Thus, we concluded that aggravated cerebral mitochondria injury might be attributed to CSD under hypoxic conditions. Flunarizine can alleviate such
\end{abstract}

F. Li · E. Qiu · Z. Dong · R. Liu · S. Wu · S. Yu ( $₫)$ Department of Neurology, Chinese PLA General Hospital, Beijing 100853, China

e-mail: yusy1963@126.com cerebral mitochondria injury under both normoxic and hypoxic conditions.

Keywords Cortical spreading depression . Mitochondria · Flunarizine $\cdot$ Hypoxia

\section{Introduction}

Cortical spreading depression (CSD) is a self-propagating wave of cellular depolarization that has been implicated in migraine and in progressive neuronal injury after stroke and head trauma.CSD which was first described by Leao [1], comprises a wave of reversible EEG suppression that propagates at a rate of $2-3 \mathrm{~mm} / \mathrm{min}$ across the cortical surface, accompanied by a negative deflection of the direct current (DC) potential and a severe disruption in ion homeostasis $[2,3]$. Recovery from CSD depends upon ion pump activity to cause an increase in metabolic activity and oxygen demand [4-6]. In normal brain tissue, this chain of events is partially compensated by an increase in cerebral blood supply [6, 7]. This coupling will be disturbed in tissues which lack oxygen. Mitochondria are key to both apoptotic and necrotic brain cell death. Several prominent mitochondrial alterations, including changes in mitochondrial respiratory function and mitochondrial membrane potential (MMP) [8], accompanied by hypoxia can contribute to cell death. A variety of electrophysiological, visual and psychological events have indicated that CSD-like events occur during the initiation of migraines [9-13], and usually, but not always, a cerebral oligemia is the only cerebral vascular response consistently detected in these circumstances [14-16]. Cellular hypoxia, in turn, can cause an increase in the flow of calcium from the extracellular fluid to the intracellular space, resulting in calcium 
overload and cellular dysfunction. The piperazine derivative flunarizine, a non-selective calcium antagonist [17], shown in many controlled clinical studies to be effective in migraine prophylaxis [18-26], has been found to protect brain tissue against hypoxic damage [27]. Therefore, the purpose of this study is to explore whether cerebral mitochondrial injury is related to CSD under both normoxic and hypoxic conditions and to find whether flunarizine has a protective effect on cerebral mitochondria.

\section{Methods}

This study on animals complies with the prevailing standards of animal welfare embodied in UK laws governing animal experimentation.

\section{Rats' surgical preparation}

CSD male rats (220-250 g) were placed in individual cages in a thermoregulated $\left(22^{\circ} \mathrm{C}\right), 12 \mathrm{~h}$ night/12 h day room. They were allowed free access to water but fasted for $12-16 \mathrm{~h}$ before surgery. Rats $(n=58)$ were anesthetized with urethane via a $20 \%$ oxygen-balance nitrogen mixture. Then they were warmed by a heating lamp $\left(37 \pm 0.5^{\circ} \mathrm{C}\right)$ above their dorsal surface during surgical procedures and controlled via a rectal temperature probe. A tail artery was cannulated for sampling blood and monitoring arterial blood pressure.

A 2-3 $\mathrm{mm}$ left parasagittal skin incision was made from the midline. The wound edges were spread and the skull bone scraped. Then two small (1-2 mm diameter) craniotomies were drilled. The former was $2 \mathrm{~mm}$ lateral and $3 \mathrm{~mm}$ anterior to bregma, while the latter was $6 \mathrm{~mm}$ posterior and $5 \mathrm{~mm}$ lateral to bregma [28]. Both craniotomies were carefully drilled so that the underlying dura would not be torn. CSD was induced by microinjection of $\mathrm{KCl}$ via a microinjector placed $1 \mathrm{~mm}$ below the pial surface of the posterior craniotomy. $0.5 \mu \mathrm{KCl}(1 \mathrm{M})$ was injected every 10 min within $1 \mathrm{~h}$. Rats of Group II or Group V were injected with $\mathrm{NaCl}(1 \mathrm{M})$ at an analogous dose and frequency. A glass micropipette (tip diameter, $1 \mu \mathrm{m}$; filled with $0.5 \mathrm{M}$ sodium acetate) was positioned $1 \mathrm{~mm}$ below the pial surface through the anterior craniotomy to monitor DC neocortical potentials and to confirm whether CSD occurred. Microelectrodes were connected to an amplifier system, and then DC signals were displayed on a chart recorder. An indifferent electrode was positioned in the left neck muscle.

Animal groups

Fifty-six SD rats were divided into seven groups randomly with each group consisting of eight rats. Group I was the control group. Group II received $1 \mathrm{M} \mathrm{NaCl}$ injections to the left parietal cortex. Group III received $1 \mathrm{M} \mathrm{KCl}$ injections to the left parietal cortex. Group IV received an intraperitoneal dose of flunarizine $(3 \mathrm{mg} / \mathrm{kg}$ ) $30 \mathrm{~min}$ before $\mathrm{KCl}$ injections mentioned above in Group III. Group V inhaled $14 \% \mathrm{O}_{2}$ for $30 \mathrm{~min}$ before $1 \mathrm{M} \mathrm{NaCl}$ injections. Group VI inhaled $14 \% \mathrm{O}_{2}$ for $30 \mathrm{~min}$ before $1 \mathrm{M} \mathrm{KCl}$ injections. Group VII inhaled $14 \% \mathrm{O}_{2}$ for $30 \mathrm{~min}$ and received an intraperitoneal dose of flunarizine $(3 \mathrm{mg} / \mathrm{kg})$ $30 \mathrm{~min}$ before the $1 \mathrm{M} \mathrm{KCl}$ injections.

\section{Mitochondrial oxidative respiratory function and MMP}

Fresh mitochondria were isolated from cerebral cortex as described by Clark [29]. The brain was placed in an ice-cold isolation media containing $0.25 \mathrm{M}$ sucrose, $0.5 \mathrm{mM}$ EDTA (dipotassium salt) and Tris- $\mathrm{HCl}(\mathrm{pH} 7.4)$ immediately after the removal. The cerebral cortex was finely minced with scissors and homogenized with isolation buffer in an allglass Teflon homogenizer. The homogenate was centrifuged at $2,000 \times g$ for $3 \mathrm{~min}$, and the supernatant was carefully decanted and saved. The pellet was resuspended in half of the original volume of isolation buffer and centrifuged in the way mentioned above. The supernatant was saved and the pellet was discarded. The pooled supernatants were centrifuged at $12,500 \times g$ for $8 \mathrm{~min}$. The resulting pellet was resuspended in 3\% Ficoll solution, layered in $6 \%$ Ficoll solution, and then centrifuged at $11,500 \times g$ for $30 \mathrm{~min}$. The pellet was resuspended in an appropriate volume of isolation buffer, and centrifuged at $12,000 \times g$ for $8 \mathrm{~min}$. All the procedures were completed at $0-4^{\circ} \mathrm{C}$ within $90 \mathrm{~min}$. The concentration of mitochondrial protein was measured by the Lowry method. The purified mitochondrial fraction was used for mitochondrial respiration and mitochondrial membrane potential measurements.

Oxygen uptake of isolated mitochondria $\left(\mathrm{nmol} \mathrm{O}_{2} / \mathrm{min}\right.$ per mg protein) was determined by polarography using a Clark-type oxygen electrode at $28^{\circ} \mathrm{C}$ as described by Chávez [30]. The pellet of mitochondria obtained from final centrifugation was resuspended and transferred to the oxygen electrode chamber in $800 \mu \mathrm{l}$ buffer which contained $100 \mathrm{mM} \mathrm{KCl}, 75 \mathrm{mM}$ mannitol, $25 \mathrm{mM}$ sucrose, $5 \mathrm{mM}$ potassium phosphate, 0.05 EDTA $\left(\mathrm{K}^{+}\right.$salt $)$and $10 \mathrm{mM}$ Tris- $\mathrm{HCl}$ (pH 7.4). $5 \mathrm{mM}$ pyruvate plus $2.5 \mathrm{mM}$ malate were included as substrates. Incubation conditions for determining state 3 (ST3) (ADP and substrates present) and state 4 respiration (ST4) (after ADP was depleted) were as defined by Chance and Williams [31]. Respiration control rate (RCR) is defined as ST3 divided by ST4.

Hundred microliter of mitochondria obtained from final centrifugation, $2.5 \mu \mathrm{M}$ rotenone and $1 \mu \mathrm{M}$ rhodamine 123 were resuspended and transferred to $1 \mathrm{ml}$ of reaction media which contained $150 \mathrm{mM}$ sucrose, $5 \mathrm{mM}$ sodium 
succinate, $5 \mathrm{mM} \mathrm{K} \mathrm{K}_{3} \mathrm{PO}_{4}, 20 \mathrm{mM}$ HEPES and $5 \mathrm{mM}$ $\mathrm{MgCl}_{2} .5 \mathrm{~min}$ later, the mixed solution was centrifuged at $5,000 \times g$ for $5 \mathrm{~min}$ at room temperature.

[Rhodamine 123$]_{\text {out }}$ was made with a spectrophotometer at $500 \mathrm{~nm}$. Membrane potentials (negative inside) were calculated by the Nernst equation: Mitochondria membrane potentials $\quad(\mathrm{MMP})=59 \log \left([X]_{\text {in }} /[X]_{\text {out }}\right)$. [Rhodamine $123]_{\text {in }}$ was estimated from rhodamine 123 uptake assuming distribution into a matrix space of $1 \mu \mathrm{l} / \mathrm{mg}$ of protein [32].

\section{Statistical analysis}

The data of duration of CSD in each group are expressed as median $25 \%$ quartile to $75 \%$ quartile, and statistical analysis is performed using $\mathrm{K}$ related samples test. The other data are expressed as means \pm SEM, and statistical analysis is performed using ANOVA, followed by post hoc Fisher's test. Software SPSS 13.0 is used. Significance is assumed at $P<0.05$.

\section{Results}

Cortical spreading depression

About 3 min after $\mathrm{KCl}$ injection, CSD was induced in rats of Group III, Group IV, Group VI and Group VII (see Fig. 1). The manifestation of CSD in Group III was an extracellular negative potential with amplitude of $10-30 \mathrm{mV}$ and duration of $0.5-1.5 \mathrm{~min}$, which may be preceded or succeeded by a positive deflection of variable amplitude and duration. The manifestation of CSD in Group IV, Group VI, and Group VII was similar to that in Group III. There was no CSD recorded in Group II and Group V.

The duration of CSD in Group $\mathrm{V}$ was significantly longer than that in Group III $(P<0.01)$. The number of CSD in Group IV was significantly less than that in Group III $(P<0.01)$. The amplitude of CSD in Group IV was significantly smaller than that in Group III $(P<0.05)$. The duration of CSD in Group IV was significantly shorter than that in Group III $(P<0.01)$. The number of CSD in Group VII was significantly less than that in Group VI $(P<0.05)$ (Table 1). The duration of CSD in Group VII was significantly shorter than that in Group VI $(P<0.01)$ (Table 2).

Mitochondrial oxidative respiratory function

ST3 in Group VI was significantly lower than that in Group I and Group II $(P<0.01)$. ST3 in Group VII was significantly higher than that in Group VI $(P<0.05)$. ST4 in Group VI was significantly higher than that in Group I and Group II $(P<0.01)$. RCR of Group VI was significantly

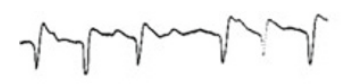

Group III

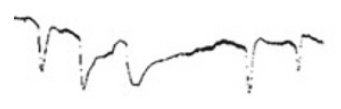

Group VI

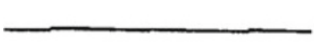

Group II

Group V

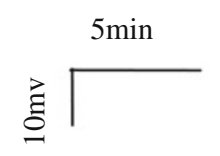

Fig. 1 The manifestation of CSD

Table 1 The number and amplitude of CSD (mean \pm SD)

\begin{tabular}{lll}
\hline Group & Number & Amplitude (mv) \\
\hline Group III & $9.25 \pm 3.81$ & $14.54 \pm 6.05$ \\
Group IV & $3.88 \pm 4.05^{* *}$ & $11.75 \pm 4.13^{*}$ \\
Group VI & $8.63 \pm 2.67$ & $16.91 \pm 6.12$ \\
Group VII & $5.00 \pm 3.16^{*, \Delta}$ & $15.29 \pm 3.39$ \\
\hline
\end{tabular}

$* P<0.05$ versus Group III; $* * P<0.01$ versus Group III;

${ }^{\Delta} P<0.05$ versus Group VI

Table 2 The duration of CSD in each group

\begin{tabular}{ll}
\hline Group & Duration (s) \\
\hline Group III & $59(49.5-72.5)$ \\
Group IV & $55(50-62)^{*}$ \\
Group VI & $75(64-92)^{* *}$ \\
Group VII & $65(58.5-79)^{* *, \Delta \Delta}$ \\
\hline
\end{tabular}

The values are median (25\% quartile-75\% quartile)

$* P<0.05$ versus Group III; $* * P<0.01$ versus Group III; ${ }^{\Delta \Delta}$ $P<0.01$ versus Group VI 
Table 3 Mitochondrial oxidative respiratory function (mean \pm SD)

\begin{tabular}{|c|c|c|c|}
\hline Group & ST3 (nmol/min/mg pro) & ST4 (nmol/min/mg pro) & $\mathrm{RCR}$ \\
\hline Group I & $103.05 \pm 21.73$ & $18.29 \pm 7.15$ & $6.18 \pm 2.02$ \\
\hline Group II & $102.27 \pm 8.88$ & $18.36 \pm 5.66$ & $6.04 \pm 2.05$ \\
\hline Group III & $82.47 \pm 24.00$ & $29.26 \pm 12.12$ & $3.14 \pm 1.46$ \\
\hline Group IV & $89.39 \pm 33.95$ & $29.16 \pm 17.61$ & $4.54 \pm 1.06$ \\
\hline Group V & $93.35 \pm 22.02$ & $43.05 \pm 16.68^{* *, \Delta \Delta}$ & $3.30 \pm 1.44$ \\
\hline Group VI & $61.73 \pm 23.44^{* *}, \mathbf{\Delta \Lambda}$ & $45.66 \pm 18.11^{* *, \diamond}$ & $1.36 \pm 0.35^{* *}$ \\
\hline Group VII & $86.28 \pm 19.28^{\text {级 }}$ & $37.62 \pm 11.19 * *$ & $2.34 \pm 0.36^{* \text {, 许岤 }}$ \\
\hline
\end{tabular}

$* P<0.05$ versus Group I; ** $P<0.01$ versus Group I; ${ }^{\Delta \Delta} P<0.01$ versus Group II; ${ }^{\diamond} P<0.05$ versus Group III; ${ }^{\mathbf{\Lambda}} P<0.01$ versus Group

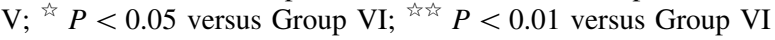

Table 4 MMP in each group (mean $\pm \mathrm{SD}$ )

\begin{tabular}{|c|c|}
\hline Group & MMP (mv) \\
\hline Group I & $194.30 \pm 10.58$ \\
\hline Group II & $188.94 \pm 7.25$ \\
\hline Group III & $161.89 \pm 14.99^{* *, \Delta \Delta}$ \\
\hline Group IV & $179.06 \pm 11.60^{*}, \diamond \diamond$ \\
\hline Group V & $182.59 \pm 8.21$ \\
\hline Group VI & $144.78 \pm 19.20^{* *}, \diamond \diamond, \boldsymbol{\Lambda}$ \\
\hline Group VII & 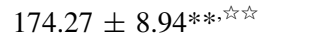 \\
\hline
\end{tabular}

$* P<0.05$ versus Group I; $* * P<0.01$ versus Group I; ${ }^{\Delta \Delta} P<0.01$ versus Group II; ${ }^{\diamond} P<0.01$ versus Group III; ${ }^{\mathbf{\Delta}} P<0.01$ versus Group V; $P<0.01$ versus Group VI

lower than that in Group I $(P<0.01)$. RCR of Group VII was significantly higher than that in Group VI $(P<0.01)$ (Table 3).

\section{MMP}

MMP in Group III $(P<0.01)$ and Group VI $(P<0.05)$ was significantly lower than that in Group I. MMP in Group VI was significantly lower than that in Group $\mathrm{III}(P<0.01)$. MMP in Group IV was significantly higher than that in Group III $(P<0.01)$. MMP in Group VII was significantly higher than that in Group VI $(P<0.01)$ (Table 4).

\section{Discussion}

CSD is now recognized to be probably the neurophysiological substrate of classical migraine aura, and may be involved in migraines without a perceived aura as well, although it has also been a focus of intense investigation in relation to apparently diverse disease states including ischemic or hemorrhagic stroke and head trauma [33]. Several recent descriptions of CSD in humans in the setting of brain injury provide definitive evidence that this phenomenon can occur and have important functional consequence in the human brain. Mayevsky and colleagues [34] used an invasive multiparametric monitoring system to record reduction of electroencephalogram (EEG) amplitude, and an increase in extracellular $\mathrm{K}^{+}$in a patient with severe head injury. Strong and colleagues [35] used electrodes placed near foci of damaged cortical tissue in patients with intracranial or subarachnoid hemorrhage to record propagated depression of electrocorticographic (ECoG) activity whose rate of spread was the same as that of CSD. Despite the longstanding assumption that CSD is the primary mechanism underlying the migraine aura, there has yet been no definitive demonstration of the electrophysiological features of CSD in a migraine patient. The spatial and temporal characteristics of migraine aura symptoms and of the propagated changes in blood flow and functional MRI signal observed in migraine patients are certainly consistent with CSD [36]. However, surface EEG recording in migraine patients, including a few documented patients with migraine with aura, have not demonstrated the classical electrophysiological features of CSD [37]. This may be because surface EEG does not have the sensitivity to detect CSD. Recent studies suggest that spreading depression could be the final common pathophysiological target for several established or investigational migraine prophylactic drugs [33]. Five clinically effective migraine prophylactic drugs (valproate, topiramate, propranolol, amitriptyline, methysergide) were shown to suppress SD susceptibility [38]. Flunarizine showed a somewhat dose-dependent CSD suppression [39].

The number of CSD represents the susceptibility of the cortex to CSD. In this study, the number of CSD under both normoxic and hypoxic conditions was examined. Decreasing the percentage of $\mathrm{O}_{2}$ from room air concentration $(21 \%)$ to $14 \%$ did not change the number of CSD. Takano [6] observed that decreasing the percentage of $\mathrm{O}_{2}$ from room air concentration $(21 \%)$ to $8 \%$ generated CSD without $\mathrm{KCl}$ injection. From the two experiments, we concluded that the susceptibility of cortex to CSD increased at $8-14 \% \mathrm{O}_{2}$. The susceptibility of the cortex to CSD is also affected by many other factors including 
cytoarchitecture, maturity and distribution of transmitter systems, ion channels in the neuron membrane and $\mathrm{pH}$ [40].

The wave of CSD is produced by neuron dendritic persistent inward currents. The amplitude of CSD is regulated by glutamate-controlled channels, sodium channels and calcium channels [41, 42]. In the present study, the amplitude of CSD under normoxic and hypoxic conditions was examined. Decreasing the percentage of $\mathrm{O}_{2}$ from room air concentration $(21 \%)$ to $14 \%$ did not change the amplitude of CSD. Therefore, we concluded that the amplitude of CSD was not affected by $\mathrm{O}_{2}$ concentration.

The duration of CSD is related to the repolarization of neurons and glial cells. During this study, we found an inverse correlation between $\mathrm{O}_{2}$ supply and the duration of CSD. The finding implied that $\mathrm{O}_{2}$ supply was the important factor for normalization of extracellular $\mathrm{K}^{+}$.

Flunarizine is reported to be the only calcium antagonist capable of protecting brain cells against hypoxic damage $[42,43]$. Results of more than 10 open and almost 20 placebo-controlled trials of migraine prophylaxis with flunarizine demonstrate the beneficial effects and safety of this agent [18]. Meanwhile, eight clinical trials compared the calcium channel blocker with $\beta$-adrenoceptor blockers (six trials with propranolol, two with metoprolol) [19-26], in which flunarizine was equally effective with the $\beta$-adrenoceptor blockers, but had a qualitatively different adverse event profile. Hence, flunarizine is considered a drug of first choice in migraine prophylaxis, and is used as first-line prophylactic agents in most European countries [44].

In the present study, it was found that flunarizine decreased the number and amplitude of CSD, and shortened the duration of CSD under normoxic conditions. Also it was found that flunarizine decreased the number and duration of CSD under hypoxic conditions.

Flunarizine reduced the susceptibility of the cortex to CSD under normoxic and hypoxic conditions. Blockers of $\mathrm{L}-, \mathrm{N}-$, and $\mathrm{P} / \mathrm{Q}-$ type voltage-gated calcium channels decreased the number of CSD [45]. These results suggested the effects of flunarizine on the number of CSD can be attributed to its blockers of L-, N-type voltage-gated calcium channels.

Flunarizine shortened the duration of CSD under both normoxic and hypoxic conditions, which may be attributed to its inhibitory effect on cortical hypoperfusion induced by CSD [46]. Thus, $\mathrm{O}_{2}$ supply in brain tissue is increased, which allowed the repolarization of neurons and glial cells.

Flunarizine decreased the amplitude of CSD under normoxic conditions, which may be attributed to the amplitude of CSD regulated by calcium antagonist [40]. Flunarizine did not decrease the amplitude of CSD under hypoxic conditions. This finding indicated that hypoxia attenuated the effect of calcium antagonists on the amplitude of CSD.
Whether CSD can cause irreversible neuronal injury or not is uncertain. As mentioned by Nedergaard and Hansen [47], in normally circulated and oxygenated healthy adult brains, SD can be provoked many times without obvious harm. Pomper et al. [48] report that repeated episodes of spreading depression-like events (SDLEs) caused irreversible injury in hippocampal slice cultures taken from immature brains. Mitochondria have many functions in eukaryotic cells. Perhaps the most important one is the generation of adenosine $5^{\prime}$-triphosphate (ATP) by oxidative phosphorylation. Since it had been shown that tissue ATP content decreases to $54 \%$ in rats during the passages of SD waves, the capability of tissue energy production has been studied [49]. The mitochondrial redox state inclines to the reduction side synchronous to the onset of CSD and normalizes within $120 \mathrm{~s}$ after the onset of depolarization by measuring the NADH fluorescence in vivo [50]. Along with many mitochondrial carrier-mediated processes, the generation of ATP requires an electrochemical potential generated by the electron transport chain. Thus, normally functioning mitochondria establish MMP and a $\mathrm{pH}$ gradient $(\Delta \mathrm{pH})$, which jointly comprises $\Delta p$, the proton motive force that drives ATP synthesis. Processes that dissipate $\Delta p$ are usually considered harmful to cells. MMP is typically the more dynamic parameter in the proton motive force. MMP represents a common final pathway of many conditions associated with oxidative stress including hypoxia, hypoglycemia, and aging. MMP dissipation may be caused by ATP synthesis, $\mathrm{Ca}^{2+}$ transport, or the activity of other carrier proteins [51]. In this study, MMP dissipated due to CSD and further dissipated due to CSD under hypoxic conditions. Thus, our data indicated that CSD caused oxidative stress or aggravated hypoxic conditions in the brain. These changes can be attenuated by flunarizine.

ST3 reflects the maximum rate of coupled respiration, that is, when electron transport is coupled to ATP synthesis. ST4 reflects the rate of leakage of protons back across the inner mitochondrial membrane into the matrix. RCR reflects the coupling rate of oxidation phosphorylation. Our data indicated that CSD under hypoxic conditions decreased the maximal rate of coupled respiration, while increased the rate of leakage of protons back across the inner mitochondrial membrane into the matrix, and further uncoupled the mitochondria. The change of mitochondrial oxidative respiratory function by CSD under hypoxic conditions can partly be attenuated by flunarizine.

The exact pathogenesis of migraine is not determined and may be polymorphic. Platelet mitochondrial enzyme activities study has shown that NADH-dehydrogenase, citrate synthase and cytochrome $c$ oxidase activities are significantly lower in migraineurs than in controls [52]. A deficient energy metabolism could be the missing link between the biobehavioral model and the hypoxia theory of migraine. It 
would explain why an excessive activation of cortical structures could lead to trigeminovascular activation via a metabolic disequilibrium, and possibly via hypoxia-sensing neurons and the hypoxia-inducible factor [53].

In conclusion, our work indicated that aggravated cerebral mitochondria injury might be induced by CSD under hypoxic conditions, and that flunarizine can alleviate cerebral mitochondria injury under both normoxic and hypoxic conditions. However, our work is limited to experimental animals and any link to migraine pathophysiology is purely hypothetical.

Acknowledgments Supported by the 11th 5-year plan of PLA (06MA256).

Conflict of interest We declare that we have no financial and personal relationships with other people or organizations that can inappropriately influence our work, there is no professional or other personal interest of any nature or kind in any product, service and/or company that could be construed as influencing the position presented in the manuscript entitled "Protection of Flunarizine on Cerebral Mitochondria Injury Induced by Cortical Spreading Depression under Hypoxic Condition".

Open Access This article is distributed under the terms of the Creative Commons Attribution License which permits any use, distribution and reproduction in any medium, provided the original author(s) and source are credited.

\section{References}

1. Leao AAP (1944) Spreading depression of activity in cerebral cortex. J Neurophysiol 7:359-390

2. Haghir H, Kovac S, Speckmann EJ, Zilles K, Gorji A (2009) Patterns of neurotransmitter receptor distributions following cortical spreading depression. Neuroscience 163:1340-1352

3. Chang JC, Shook LL, Biag J, Nguyen EN, Toga AW, Charles AC, Brennan KC (2010) Biphasic direct current shift, haemoglobin desaturation and neurovascular uncoupling in cortical spreading depression. Brain 133:996-1012

4. Piilgaard H, Lauritzen M (2009) Persistent increase in oxygen consumption and impaired neurovascular coupling after spreading depression in rat neocortex. J Cereb Blod Flow Metab 29:1517-1527

5. Funke F, Kron M, Dutschmann M, Müller M (2009) Infant brain stem is prone to the generation of spreading depression during severe hypoxia. J Neurophysiol 101:2395-2410

6. Takano T, Tian GF, Peng W, Lou N, Lovatt D, Hansen AJ, Kasischke KA, Nedergaard M (2007) Cortical spreading depression causes and coincides with tissue hypoxia. Nat Neurosci 10:754-762

7. Sonn J, Mayevsky A (2000) Effects of brain oxygenation on metabolic, hemodynamic, ionic and electrical responses to spreading depression in the rat. Brain Res 882:212-216

8. Ouyang YB, Xu LJ, Sun YJ, Giffard RG (2006) Overexpression of inducible heat shock protein 70 and its mutants in astrocytes is associated with maintenance of mitochondrial physiology during glucose deprivation stress. Cell Stress Chaperones 11:180-186

9. Bartolini M, Baruffaldi R, Paolino I, Silvestrini M (2005) Cerebral blood flow changes in the different phases of migraine. Funct Neurol 20:209-211
10. Lauritzen M, Olsen TS, Lassen NA, Paulson OB (1983) Regulation of regional cerebral blood flow during and between migraine attacks. Ann Neurol 14:569-572

11. Lauritzen M (2001) Cortical spreading depression in migraine. Cephalalgia 21:757-760

12. Dalkara T, Zervas NT, Moskowitz MA (2006) From spreading depression to the trigeminovascular system. Neurol Sci 27:S86S90

13. Mayevsky A, Doron A, Manor T, Mellin S, Zarchin N, Ouaknine GE (1996) Cortical spreading depression recorded from the human brain using a multiparametric monitoring system. Brain Res 740:268-274

14. Lauritzen M, Olsen TS, Lassen NA, Paulson OB (1983) Changes in regional cerebral blood flow during the course of classic migraine attacks. Ann Neurol 13:633-641

15. Olesen J, Larsen B, Lauritzen M (1981) Focal hyperemia followed by spreading oligemia and impaired activation of $\mathrm{rCBF}$ in classic migraine. Ann Neurol 9:344-352

16. Woods RP, Iacoboni M, Mazziotta JC (1994) Brief report: bilateral spreading cerebral hypoperfusion during spontaneous migraine headache. N Engl J Med 331:1689-1692

17. Gelmers HJ (1985) Calcium-channel blockers in the treatment of migraine. Am J Cardiol 55:139B-143B

18. Reveiz-Herault L, Cardona AF, Ospina EG, Carrillo P (2003) Effectiveness of flunarizine in the prophylaxis of migraine: a meta-analytical review of the literature. Rev Neurol 36:907-912

19. Grotemeyer KH, Schlake HP, Husstedt IW (1988) Prevention of migraine with metoprolol and flunarizine. A double-blind crossover study. Nervenarzt 59:549-552

20. Ludin HP (1989) Flunarizine and propanolol in the treatment of migraine. Headache 29:219-224

21. Shimell CJ, Fritz VU, Levien SL (1990) A comparative trial of flunarizine and propranolol in the prevention of migraine. S Afr Med J 77:75-77

22. Lutschg J, Vassella F (1990) The treatment of juvenile migraine using flunarizine or propranolol. Schweiz Med Wochenschr 120:1731-1736

23. Sørensen PS, Larsen BH, Rasmussen MJ, Kinge E, Iversen H, Alslev T, Nøhr P, Pedersen KK, Schrøder P, Lademann A (1991) Flunarizine versus metoprolol in migraine prophylaxis: a doubleblind, randomized parallel group study of efficacy and tolerability. Headache 31:650-657

24. Gawel MJ, Kreeft J, Nelson RF, Simard D, Arnott WS (1992) Comparison of the efficacy and safety of flunarizine to propranolol in the prophylaxis of migraine. Can J Neurol Sci 19:340-345

25. Verspeelt J, Locht P, Amery WK (1996) Post-marketing cohort study comparing the safety and efficacy of flunarizine and propranolol in the prophylaxis of migraine. Cephalalgia 16:328-336

26. Bordini CA, Arruda MA, Ciciarelli MC, Speciali JG (1997) Propranolol vs flunarizine vs flunarizine plus propranolol in migraine without aura prophylaxis. A double-blind trial. Arq Neuropsiquiatr 55:536-541

27. Holmes B, Brogden RN, Heel RC, Speight TM, Avery GS (1984) Flunarizine a review of its pharmacodynamic and pharmacokinetic properties and therapeutic use. Drugs 27:6-44

28. Moskowitz MA, Nozaki K, Kraig RP (1993) Neocortical spreading depression provokes the expression of $\mathrm{c}$-fos proteinlike immunoreactivity within trigeminal nucleus caudalis via trigeminovascular mechanisms. J Neurosci 13:1167-1177

29. Clark JB, Nicklas WJ (1970) The metabolism of rat brain mitochondria. J Biol Chem 245:4724-4731

30. Chávez JZ, Pichiule P, Boero J, Arregui A (1995) Reduced mitochondrial respiration in mouse cerebral cortex during chronic hypoxia. Neurosci Lett 193:169-172

31. Chance B, Williams GR (1956) The respiratory chain and oxidative phosphorylation. Adv Enzymol 17:65-134 
32. Emaus RK, Grunwald R, Lemasters JJ (1986) Rhodamine 123 as a probe of transmembrane potential in isolated rat-liver mitochondria: spectral and metabolic properties. Biochim Biophys Acta 850:436-448

33. Ayata C (2009) Spreading depression: from serendipity to targeted therapy in migraine prophylaxis. Cephalalgia 29:1097-1114

34. Mayevsky A, Doron A, Manor T, Meilin S, Zarchin N, Ouaknine GE (1996) Cortical spreading depression recorded from the human brain using a multiparametric monitoring system. Brain Res 740:268-274

35. Strong AJ, Fabricius M, Boutelle MG, Hibbins SJ, Hopwood SE, Jones R, Parkin MC, Lauritzen M (2002) Spreading and synchronous depressions of cortical activity in acutely injured human brain. Stroke 33:2738-2743

36. Charles A, Brennan K (2009) Cortical spreading depression-new insights and persistent questions. Cephalalgia 29:1115-1124

37. Lauritzen M, Trojaborg W, Olesen J (1981) EEG during attacks of common and classical migraine. Cephalalgia 1:63-66

38. Ayata C, Jin H, Kudo C, Dalkara T, Moskowitz MA (2006) Suppression of cortical spreading depression in migraine prophylaxis. Ann Neurol 59:652-661

39. Wauquier A, Ashton D, Marrannes R (1985) The effects of flunarizine in experimental models related to the pathogenesis of migraine. Cephalalgia 5(Suppl 2):119-123

40. Somjen GG (2001) Mechanisms of spreading depression and hypoxic spreading depression-like depolarization. Physiol Rev 81:1065-1096

41. Wadman WJ, Juta AJA, kamphuis W, Somjen GG (1992) Current source density of sustained potential shifts associated with electrographic seizures and with spreading depression in rat hippocampus. Brain Res 570:85-91

42. Jing J, Aitken PG, Somjen GG (1993) Role of calcium channels in spreading depression in rat hippocampal slices. Brain Res 604:251-259

43. Leone M, Grazzi L, La Mantia L, Bussone G (1991) Flunuaizine in migraine: a minireview. Headache 31:388-391
44. Members of the task force, Ever S, Afra J, Frese A, Goadsby PJ, Linde M, May A, Sándor PS (2006) EFNS guideline on the drug treatment of migraine-report of an EFNS task force. Eur J Neurol 13:560-572

45. Richter F, Ebersberger A, Schaible HG (2002) Blockade of voltage-gated calcium channels in rat inhibits repetitive cortical spreading depression. Neurosci Lett 334:123-126

46. Shimazawa M, Hara H, Watano T, Sukamoto T (1995) Effects of $\mathrm{Ca} 2+$ channel blockers on cortical hypoperfusion and expression of c-Fos-like immunoreactivity after cortical spreading depression in rats. Br J Pharmacol 115:1359-1368

47. Nedergaard M, Hansen AJ (1988) Spreading depression is not associated with neuronal injury in the normal brain. Brain Res 449:395-398

48. Pomper JK, Haack S, Petzold GC, Buchheim K, Gabriel S, Hoffmann U, Heinemann U (2006) Repetitive normoxic spreading depression like events result in cell damage in juvenile hippocampal slice cultures. J Neurophysiol 95:355-368

49. Mies G, Paschen W (1984) Regional changes of blood flow, glucose, and ATP content determined on brain sections during a single passage of spreading depression in rat brain cortex. Exp Neurol 84:249-258

50. Hashimoto M, Takeda Y, Sato T, Kawahara H, Nagano O, Hirakawa M (2000) Dynamic changes of NADH fluorescence images and NADH content during spreading depression in the cerebral cortex of gerbils. Brain Res 87:294-300

51. Vergun O, Vltyakova TV, Reynolds IJ (2003) Spontaneous changes in mitochondrial membrane potential in single isolated brain mitochondria. Biophys J 85:3358-3366

52. Sangiorgi s, Mochi M, Riva R, Gortelli P, Monari L, Pierangeli G, Montagna P (1994) Abnormal platelet mitochondrial function in patients affected by migraine with and without aura. Cephalalgia 14:21-23

53. Magis D, Ambrosini A, Sándor P, Jacquy J, Laloux P, Schoenen J (2007) A randomized double-blind placebo-controlled trial of thioctic acid in migraine prophylaxis. Headache 47:52-57 